\title{
Controlled Vesicle Self-Assembly in Microfluidic Channels with Hydrodynamic
}

\section{Focusing}

Andreas Jahn*, Wyatt N. Vreeland ${ }^{\dagger}$, Michael Gaitan*, Laurie E. Locascio ${ }^{\dagger}$

*Semiconductor Electronics Division, ${ }^{\dagger}$ Analytical Chemistry Division, National Institute of

Standards and Technology Gaithersburg MD 20899

Phone: 301-975-2070; Fax: 301-977-0587; Email: michael.gaitan@nist.gov,

laurie.locascio@nist.gov

\section{Supporting Information ${ }^{1}$}

\section{Lipid and Solution Preparation}

Dimyristoylphosphatidylcholine (DMPC) and cholesterol (Sigma-Aldrich, St. Louis, MO) in a molar ratio of 1:1 were diluted in chloroform with 1 wt $\%$ of $1,1^{\prime}$-dioctadecyl-3,3,3',3' tetramethilindodicarbocyanine perchlorate $\left(\mathrm{DiIC}_{18}\right)$ added for fluorescent imaging. The chloroform solvent was evaporated under a stream of nitrogen at room temperature to form a lipid film on the bottom of a test tube. The test tube was then placed in a vacuum desiccator for at least $24 \mathrm{~h}$ to ensure dryness. The dried lipid mixture was resolubilized with $500 \mu \mathrm{L}$ dry isopropanol yielding a 10 $\mathrm{mM}$ concentration of lipid solution. Phosphate buffered saline solution (10 mM phosphate, $27 \mathrm{mM}$ potassium chloride, $137 \mathrm{mM}$ sodium chloride, $\mathrm{pH}=7.4$ ) was used as the hydration buffer.

\footnotetext{
${ }^{1}$ Certain commercial equipment, instruments, or materials are identified in this report to specify adequately the experimental procedure. Such identification does not imply recommendation or endorsement by the National Institute of Standards and Technology nor does it imply that the materials or equipment identified are necessarily the best available for the purpose.
} 


\section{Microfluidic Device Fabrication}

A microchannel network (200- $\mu \mathrm{m}$ channel width) was anisotropically etched on the surface of a silicon wafer $(<100>$ orientation, $75 \mathrm{~mm}$ diameter, $0.3 \mathrm{~mm}$ thickness, Virginia Semiconductor, Fredericksburg, VA) to a depth of $40 \mu \mathrm{m}$ through a photolithographically patterned thermal $\mathrm{SiO}_{2}$ $(100 \mathrm{~nm})$ hard mask using tetramethylammonium hydroxide (TMAH, Alfa Aesar, Ward Hill, MA) (1:2 dilution $25 \% \mathrm{w} / \mathrm{w}$ aqueous TMAH solution, $80^{\circ} \mathrm{C}$ ). The same procedure was followed to etch fluid through-holes on the back-side of the wafer that were aligned to the microchannel network in the front side. Following this, all surface oxide was removed ( $8 \%$ buffered hydrofluoric acid etch) and then reoxidized to form a $100 \mathrm{~nm}$ thick $\mathrm{SiO}_{2}$ film encapsulating the silicon substrate. Finally, a glass cover wafer (75-mm diameter, 0.1-mm thickness, Corning 7740) was anodically bonded to the front surface of the silicon wafer using a $580 \mathrm{~V}$ applied potential and heated to $400{ }^{\circ} \mathrm{C}$ to seal the microchannel network.

Reagent transfers were accomplished using gastight glass syringes interfaced to the microchannel network through capillary tubing (PEEK Tubing, Upchurch Scientific, Oak Harbor, WA) and capillary connectors (Nanoports, Upchurch Scientific, Oak Harbor, WA) that were bonded to the fluid through-holes etched in the silicon wafer. Programmable syringe pumps (Harvard Apparatus, Holliston, MA) were used to control the fluid flow rates by computer using a LabVIEW software interface. 


\section{Liposome Characterization}

The liposome formulations $(100-\mu \mathrm{L}$ sample size) were collected at each flow condition into polycarbonate cuvets. After collection, $1 \mathrm{~mL}$ of phosphate buffered saline solution (10 $\mathrm{mM})$ was added to each formulation and sealed for further characterization.

Liposome size and size distribution was characterized by laser light scattering was performed using a Coulter N4 MD submicron particle analyzer collecting scattered light at $90^{\circ}$. Confocal fluorescence imaging was performed with a Pascal confocal imaging system (Carl Zeiss, Thornwood, NY).

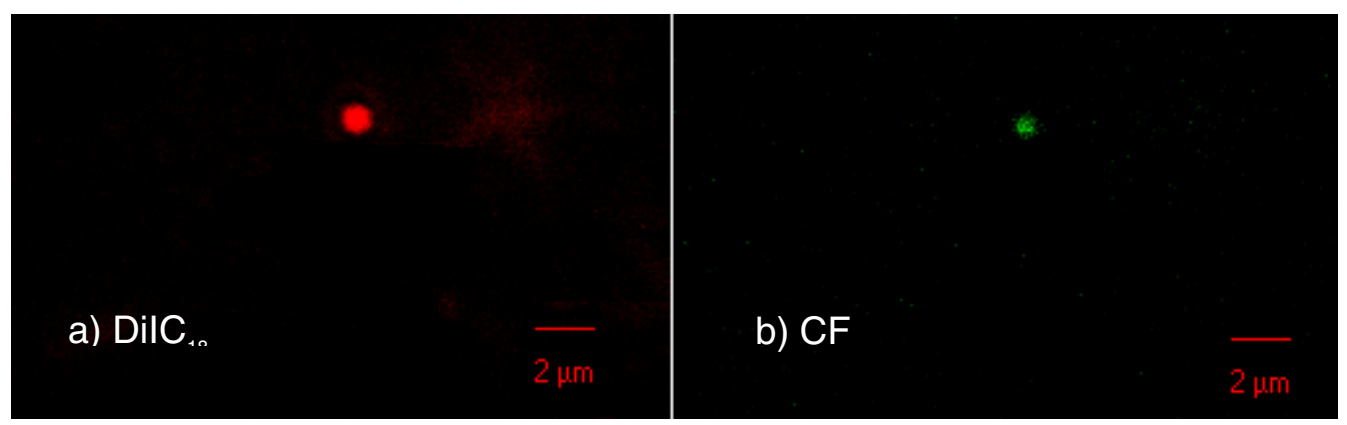

Figure S1. Two-color confocal fluorescence imaging of a single liposome formed in flow conditions of $0.5 \mu \mathrm{L} / \mathrm{min}$ flow rate of the isopropanol stream and $20 \mu \mathrm{L} / \mathrm{min}$ flow rate of the aqueous buffer streams. The flow conditions in this example produced a liposome mean diameter of $133 \mathrm{~nm}$ and standard deviation of $26 \mathrm{~nm}$ determined by light scattering measurements. (a) Fluorescence image for DiIC $_{18}$ that exhibits intercolation of the dye into the lipid bilayer; and (b) fluorescence image for carboxyfluorescein (CF) that has been encapsulated in the liposome's aqueous interior. N.B. The apparent size of the liposome in these images does not give an accurate representation of the actual liposome size due to artifacts of image processing and limitations of the imaging optics. All liposomes in our samples are below the optical resolution accessible by confocal imaging. 